\title{
STUDY ON RELATIVE ABUNDANCE OF Plasmodium SPECIES: A CASE OF PATIENTS ATTENDING JAHUN GENERAL HOSPITAL, JIAGAWA, NIGERIA.
}

\author{
Ahmed, U.A. \\ Department of Medical Laboratory Services, Jahun General Hospital, Jigawa, Nigeria. \\ umaradamuahmed@yahoo.com
}

\begin{abstract}
A study on relative abundance of Plasmodium species was conducted using blood film technique. For a 12 month period, 4037 positive blood films were exaqmined for a particular species of Plasmodium. The result revealed that 3208 (79.46\%) were Plasmodium falciparum, 157 (03.89\%) were $P$. ovale and 672 (16.65\%) were P. malariae. This showed that P. falciparum was the most abundant and the relative abundance of the species had heightened in July, 2008 due to rain which favours vector survival.
\end{abstract}

Keywords: abundance, plasmodium, relative, thin blood film, malaria control programmes.

\section{INTRODUCTION}

Plasmodium is carried by infected female Anopheles mosquito and eventually transmit it into humans through its bite. Human malaria is caused by four closely related species of Plasmodium. Which differ in their symptoms, virulence, pre-patient periods, immunological and epidemiological characteristics and sensitivity to drugs (Alnwick, 2000).

When an infected female Anopheles mosquito bites a health person it introduces parasites at the sporozoite stage into his body. These sporozoites migrates to the liver, multiply and develop into trophozoites which then invade the red blood cells. In these cells, they divide asexually forming numerous merozoites which when liberated attack many other red blood cells. Plasmodium undergoes sexual stage in humans and asexual stage in female Anopheles mosquito. Some merozoites also develop into gametocytes when it bites an infected person (Sarojini, 2007).

Four species of Plasmodium cause human malaria. They are Plasmodium vivax Grassi, 1890, P. malariae Lavera, 1897, $P$. ovale Stephens, 1922 and $P$. falciparum Welch, 1897 (Alnwick, 2000). P. malariae causes quartan malaria with a four day cycle comprising of one day fever two days of remission and another day fever (Alnwick, 2000). P. vivax causes benign tertan malaria but rarely encountered in comparison to the other three malarias. Its fevers are milder than those that caused by $P$. vivax. $P$. falciparum causes malignant tertian malaria, which is the most virulent, producing an acute course and often terminating fatally. It causes abortion and still birth (WHO, 2000).

Assessment of the Plasmodium species is crucial to the understanding of the type of infection as well as the malaria epidemiology (Crawley, 2004) for example, the type of parasite and the parasite load determine the type of treatment to be done.

\section{MATERIAL AND METHODS}

Study Area

The study area was Jahun Local Government Area of Jigawa State, Nigeria. it covers 1246.63 square kilometers of landmass. It lies between latitude $12^{\circ} 06^{\prime} \mathrm{N}$ and longitude $9^{\circ} 63 \mathrm{E}$ and is entirely in the Sudan savannah. Rain starts in June and ends in September. This study was done in the year 2009 and was localized to Jahun General Hospital. Jahun Local Government Area is inhabited predominantly by Hausas and Fulani's ethnic groups with other notable ethnic groups (Zakari, 2006).

The choice of the study site was based on the fact that the malaria cases record has shown a various among the parasite species. it was hypothesized that the highest malaria infection was due to Plasmodium falciparum species, and therefore, there is the need to clarify this variation and to verify the status of $P$. falciparum in malaria cases in the study area.

\section{Sampling Technique}

A total of 4037 blood samples of malaria positive patients were sampled and examined in twelve months according to (WHO, 2004). The lobe or the finger (or heel if it is an infant) was sterilized using a swab moistened with $70 \% \mathrm{v} / \mathrm{v}$ alcohol and the rea was allowed to dry. Using a sterile lancet, the finger or heel was pricked and squeezed gently. A small drop of blood was put at the centre of a completely clean grease - free microscope slide.

\section{Preparation of Blood Film and Staining}

Finger prick blood smears were used to prepare thin films. The slides were stained using Fields staining technique (WHO, 2004). Field stain is water-based Romanowsky stain composed of two solutions of fields stain A and fields stain B. Holding the slide with the dried thick film facing downwards, the slide was dipped into fields stain A for 5 seconds. The excess stain was drained off by touching a corner of the slide against the side of the stain container. The above was washed gently for about 5 seconds in clean water. The excess water was drained off and the slide is washed gently in clean water again, wiped at the back and placed upright in a draining rack for the film to air - dry (Moody, 2000). 


\section{Microscopy and Reporting Blood Films}

The blood films were examined microscopically using the $40 x$ and $10 x$ immersion oil objectives against $7 x$ eye piece for brighter and clearer image (WHO, 2004). The examination was made using standard keys of Edington and Gills (1976) as follows:

1. Many ring forms or crescent - shaped gametocytes indicate $P$. falciparum.

2. Parasites surrounded by pale pink semi lunar containing pale red dots indicate $P$. vivax.

3. Parasites more compact and smaller, some oval shaped parasitized cell with ragged ends indicate $P$. ovale and few thick compact rings or small round gametocytes with yellowbelow pigment indicate $P$. malariae. In this way the blood films were examined and the Plasmodium species abundance was noted.

\section{Data Analysis}

The data obtained in the study were analyzed with respect to number Plasmodium species obtained.
These were presented in tables, interpreted in percentages analyzed with respect to the number of species.

\section{RESULTS}

The annual relative abundance of Plasmodium species for the year 2009 was shown in Table 1. The total number of positive blood films were 4037, of which $P$. falciparum was the most abundant, 3208 (79.46\%), followed by $P$. malariae, $672(16.65 \%)$, then $P$. ovale, $157(03.89 \%)$ and there was no $P$. vivax reported.

The monthly abundance revealed January, 322 (7.98\%), February, 147 (3.64\%), March, 118 (2.92\%), April, 154 (3.81\%), May, 141 (3.49\%), June, 158 (3.91\%), July, 513 (12.71\%), August, 522 (12.93\%), September 514 (12.73\%), October, 542 (13.43\%), November, 495 (12.26\%) and December, $411(10.18 \%)$. This showed that the Plasmodium species were most abundant in October and $P$. falciparum was most in September, 467.

Table 1: Monthly abundance of Plasmodium species for the year 2009

\begin{tabular}{lccccc}
\hline Months & $\begin{array}{c}\text { No. of Plasmodium } \\
\text { falciparum }\end{array}$ & Plasmodium & $\begin{array}{c}\text { Plasmodium } \\
\text { ovalariae }\end{array}$ & Overall \\
\hline January & 322 & 244 & 72 & 6 & 644 \\
February & 147 & 100 & 33 & 14 & 280 \\
March & 118 & 85 & 22 & 11 & 236 \\
April & 154 & 101 & 38 & 15 & 308 \\
May & 141 & 79 & 39 & 23 & 282 \\
June & 158 & 116 & 34 & 8 & 316 \\
July & 513 & 423 & 80 & 10 & 1023 \\
August & 522 & 431 & 76 & 15 & 1044 \\
September & 514 & 467 & 34 & 13 & 1128 \\
October & 542 & 441 & 82 & 19 & 1084 \\
November & 495 & 411 & 73 & 11 & 990 \\
December & 411 & 310 & 89 & 12 & 822 \\
Relative & $\mathbf{1 0 0 . 0 0}$ & $\mathbf{7 9 . 4 6}$ & $\mathbf{1 6 . 6 5}$ & $\mathbf{0 3 . 8 9}$ & $\mathbf{1 0 0}$ \\
abundance (\%) & & & & & \\
\hline
\end{tabular}

\section{DISCUSSION}

$P$. falciparum is the most pathogenic of human malaria species with untreated infections causing severe disease and death, particularly young children, pregnant women and non-immune adults (Cheesbrough, 2005). This agreed with findings of this study where $P$. falciparum was 3208 (79.46\%).

The abundance of $P$. falciparum can be attributed to the high temperature situation of the study area been a Sudan savannah region as indicated by Blackwell (2004) that $P$. falciparum is found the hotter and more humid regions of the world.

Moreover, WHO reported that in Nigeria falciparum malaria is the most prevalent and constitutes about $100 \%$ of all infections, while quartan malaria has $15-$ $20 \%$ prevalence. Similarly, Tertian ovale malaria had the least prevalence of about $2 \%$ and occurring as mixed infection quartan and malariae infection, while the benign tertian vivax malaria is not endemic to Nigeria and West Africa (WHO, 2000). Plasmodium malariae infection is relatively rare and responsible for
$7 \%$ of malaria incidence in the world. This coincided with findings of this study where $P$. falciparum was $79.46 \%$.

$P$. malariae has much higher prevalence than $P$. ovale, $672(16.65 \%)$ and $157(0.89 \%)$ respectively. In Tropical Africa, it accounts for up to $25 \%$ of Plasmodium infections (Cheesbrough, 2005).

On the other hand, $P$. ovale was least in abundance $157(03.89 \%)$ and this had agreed with Cheesbrough (2005), that $P$. ovale has low prevalence, where it accounts for up to $10 \%$ of malaria infections in West Africa and has also been reported from other parts of Africa. And this study had not reported P. vivax, similarly, WHO (2000) reported that the vivax malaria is not endemic to Nigeria and West Africa.

The relative abundance of Plasmodium species according to months showed that October had the most abundant, 542 and this may be attributed to the fact that rain was plenty in that month and provides much more breeding sites for the Anopheles and hence Plasmodial transmission as in Service (1980); 
Anopheles mosquitoes breed in areas with water bodies which are suitable for the growth and development of various strains of mosquitoes as ponds, wells and surface water bodies of different sizes are available during rainy season which could also serve as breeding sites.

\section{CONCLUSION}

This study had indicated the abundance of Plasmodium species responsible for malaria cases in the study area namely $P$. falciparum, $P$. malariae and $P$. ovale with $P$. falciparum bearing the highest

\section{REFERENCES}

Alnwick, D. (2000). Meeting the malaria challenge Africa Health Supplement: 1819.

Blackwell, S. 92004). Malaria Lecture Notes on Tropical Medicine ( $5^{\text {th }}$ edition). Blackwell Science PP. $55-72$.

Crawley, J. (2004). Reducing the burden of anaemia in infants and young children in malaria endemic countries of Africa. From evidence to action. American Journal of Tropical Medicine and Hygiene. Supplement 2: 25 34.

Edington, G.M. and Gills, H.M. (1976). Pathology in the Tropics. Edward Arnold Publishers Ltd., United Kingdom Pp. 34.

Moody, A. (2000). Rapid diagnostic tests for malaria parasites. Clinical Microbiology Reviews. 15: 1: $66-78$.

Cheesbrough, M. (2005). District Laboratory Practice in Tropical Countries Part $12^{\text {nd }}$ edition). Cambridge University Press, Cambridge U.K., Pp $240-242$. number. This means that $P$. falciparum is responsible for most of the malaria cases among the patients attending Jahun General Hospital during the study period.

\section{Recommendation}

This study should attract multifaceted malaria control programme to include administration of Artemesinin combined theraphy (ACT), Immediate destruction of malaria vector and use of insecticide treated bed nets (ITNs).

Sarojini, T.R. (2007). Modern Biology for Senior Secondary Schools. Africana First Publishers Ltd, Ibadan, Nigeria Pp. 190.

Service, M.W. (1980). A Guide to Medical Entomology. Macmillan International College Edition. Oxford Press Ltd, Pp $23-45$.

World Health Organization (2000). WHO Expert Committee on Malaria. WHO Technical Report Series, 20 ${ }^{\text {th }}$ Report, Pp $1-29$.

World Health Organization (2004). Malaria Rapid Diagnosis. Making it work. www.who.int.rdt.

Zakari, U. (2006). Settlements and Settlers of Northern Nigeria. www. africahistory/nigeria/11/30/210. 